\title{
Observability and Geometric Approach of 2D Hybrid Systems
}

\author{
Tiberiu Vasilache \\ University "Politehnica" of Bucharest \\ Faculty of Applied Sciences \\ Splaiul Independentei 313, Ro-060032, \\ Bucharest, Romania \\ Email: tiberiu.vasilache@gmail.com
}

\author{
Valeriu Prepeliţă \\ University "Politehnica" of Bucharest \\ Faculty of Applied Sciences \\ Splaiul Independentei 313, Ro-060032, \\ Bucharest, Romania \\ Email: vprepelita@mathem.pub.ro
}

Received: May 12, 2019. Revised: July 6, 2021. Accepted: October 23, 2021. Published: November 24, 2021.

\begin{abstract}
A connection is emphasized between two branches of the Systems Theory, namely the Geometric Approach and 2D Systems, with a special regard to the concept of observability. An algorithm is provided which determines the maximal subspace which is invariant with respect to two commutative matrices and which is included in a given subspace. Observability criteria are obtained for a class of $2 \mathrm{D}$ systems by using a suitable $2 \mathrm{D}$ observability Gramian and some such criteria are derived for LTI 2D systems, as well as the geometric characterization of the subspace of unobservable states. The presented algorithm is applied to determine this subspace.
\end{abstract}

Index Terms-2D hybrid linear systems, observability, invariant subspace, geometric approach.

\section{INTRODUCTION}

Observability is a fundamental concept in Systems Theory which refers to the ability to estimate the state variable from the observation of the output determined by any control. The theory of controllability and observability has been developed in response to problems generated by technological sciences, especially in areas related to control, communication and computers. For "classical" 1D systems the observability theory was developed by Kalman [9,10], Gilbert [6], Weiss [18] etc.

In recent years the theory of two-dimensional (2D) systems became a distinct and important branch of Systems and Control Theory, due to the richness in its potential applications in various areas as digital image processing, seismic signal processing, gravity and magnetic field mapping, as well as to the abundance of non-trivial theoretical approaches. Givone and Roesser [7] [17] or Fornasini and Marchesini [4] introduced the notions of controllability and observability for their 2D models, as natural generalizations of the 1-D case. But their definitions referred to local notions which were not closely related to minimality or to canonical decomposition. Alternative definitions of modal controllability and observability based on coprimeness notions were presented in [11]; these definitions are connected to minimality.

The Geometric Approach is a trend in Systems and Control Theory developed to realize a better and neater investigation of the structural properties of the linear dynamical systems and to provide elegant solutions of problems of controller synthesis such as decoupling and pole-assignment problems for linear time-invariant multivariable systems. The Geometric Approach leads to a very clear geometric conditions for controllability, reachability, observability, constructibility and minimality of linear systems. The cornerstone of this approach is the concept of invariance of a subspace with respect to a linear transformation.

The history of the Geometric Approach starts in 1969 when Basile and Marro [2] introduced and studied the basic geometric tools called controlled and conditioned invariant subspaces which were applied to disturbance rejection or unknown-input observability and later on to the robust controlled invariant and the emphasis of the duality [3], [13]. Wonham's book [19] imposed the name of "(A,B)-invariant" instead of "(A,B)controlled invariant". The LQ problem was also studied in a geometric framework by Silverman, Hautus, Willems. Further contributions are due to numerous researchers among which Anderson, Akashi, Bhattacharyya, Kucera, Malabre, Molinari, Pearson, Francis and Schumacher.

The aim of this paper is to emphasize the possible interactions between the two directions of the Systems Theory, concerning the observability of a class of $2 \mathrm{D}$ hybrid linear systems, which is the continuous-discrete counterpart of Attasi's discrete 2D model [1]

Such systems appear in various problems like the iterative learning control synthesis [12] or repetitive processes [5]. For Roesser or Fornasini - Marchesini models, corresponding hybrid systems were studied by Kaczorek [8].

The advantage of the considered class of 2D systems, studied by Prepeliţă et al. in [14], [15] and [16], is the possibility to keep the global character of the natural generalizations of the concepts of observability and controllability, which remain related to minimality and state decomposition.

This paper provides in Section 2 an algorithm which determines the maximal subspace which is invariant with respect to two commutative matrices and included in a given subspace.

The state space representation of the considered class of systems is given in Section 3 and the state and output formulæ for these systems are deduced.

Section 4 is devoted to the study of the observability of 2D time varying systems. This property is characterized by means of a suitable 2D observability Gramian. For completely 
observable systems a formula is derived which provides the initial state by knowing the control and corresponding output.

Section 5 studies the time-invariant 2D systems and a list of observability criteria is established. A geometric characterization of the subspace of unobservable states is given in terms of invariant subspaces included in the kernel of the output matrix.

In Section 6 the algorithm presented in Section 2 is used to determine the subspace of unobservable states. A MATLAB Program and an example illustrate the proposed algorithm.

\section{MAXIMAL INVARIANT SUBSPACES WITH RESPECT TO} TWO COMMUTATIVE MATRICES

Let $\mathbf{K}$ be a field and $A_{1}, A_{2} \in \mathbf{K}^{n \times n}$ commutative matrices.

Definition 2.1: A subspace $\mathcal{V}$ of $\mathbf{K}^{n}$ is said to be $\left(A_{1}, A_{2}\right)$ invariant if

$$
\forall v \in \mathcal{V}, A_{1} v \in \mathcal{V} \text { and } A_{2} v \in \mathcal{V}
$$

Let $\mathcal{C}$ be a proper subspace of $\mathbf{K}^{n}$. The sum of the $\left(A_{1}, A_{2}\right)$-invariant subspaces included in $\mathcal{C}$ is called the maximal $\left(A_{1}, A_{2}\right)$-invariant subspace included in $\mathcal{C}$. We denote it $\max I\left(A_{1}, A_{2} ; \mathcal{C}\right)$.

For a subspace $\mathcal{V}$ of $\mathbf{K}^{n}$, we consider the subspaces $A_{1}^{-k} A_{2}^{-l} \mathcal{V}=\left\{v \in \mathbf{K}^{n} \mid A_{1}^{k} A_{2}^{l} v \in \mathcal{V}\right\}, k, l \in \mathbf{N}, A_{1}^{-0} A_{2}^{-0} \mathcal{V}=$ $\mathcal{V}$. If $v \in A_{i}^{-j} \mathcal{V}$, then $A_{i} v \in A_{i}^{-(j-1)} \mathcal{V}, i=1,2, \forall j \geq 0$.

Proposition 2.1: The maximal $\left(A_{1}, A_{2}\right)$-invariant subspace included in $\mathcal{C}$ is

$$
\max I\left(A_{1}, A_{2} ; \mathcal{C}\right)=\bigcap_{i=0}^{\infty} \bigcap_{j=0}^{\infty} A_{1}^{-i} A_{2}^{-j} \mathcal{C}
$$

Proof: Let us denote by $\mathcal{U}$ the subspace $\bigcap_{i=0}^{\infty} \bigcap_{j=0}^{\infty} A_{1}^{-i} A_{2}^{-j} \mathcal{C}$. If $v \in \mathcal{U}$ then $v \in A_{1}^{-i} A_{2}^{-j} \mathcal{C}, \forall i, j \in \mathbf{N}$ and also $v \in A_{1}^{-(i+1)} A_{2}^{-j} \mathcal{C}$ and $v \in A_{1}^{-i} A_{2}^{-(j+1)} \mathcal{C}$. It results that $A_{1} v \in A_{1}^{-i} A_{2}^{-j} \mathcal{C}$ and $A_{2} v \in A_{1}^{-i} A_{2}^{-j} \mathcal{C}, \forall i, j \in \mathbf{N}$, hence $A_{1} v \in \mathcal{U}$ and $A_{2} v \in \mathcal{U}$ i.e. is $\left(A_{1}, A_{2}\right)$-invariant. We can write by $(2.2) \mathcal{U}=\mathcal{C} \cap\left(\bigcap_{i=0}^{\infty} \bigcap_{j=0}^{\infty} A_{1}^{-i} A_{2}^{-j} \mathcal{C}\right)$ where $(i, j) \neq(0,0)$, hence $\mathcal{U}$ is included in $\mathcal{C}$.

Now, let $\mathcal{V}$ be an $\left(A_{1}, A_{2}\right)$-invariant subspace included in $\mathcal{C}$. Then, for any $v \in \mathcal{V}, A_{1}^{i} A_{2}^{j} v \in \mathcal{V} \subset \mathcal{C}, \forall i, j \in \mathbf{N}$, hence $v \in A_{1}^{-i} A_{2}^{-j} \mathcal{C}, \forall i, j \in \mathbf{N}$, which implies $v \in \mathcal{U}$. Therefore $\mathcal{V} \subset \mathcal{U}$, i.e. $\mathcal{U}=\bigcap_{i=0}^{\infty} \bigcap_{j=0}^{\infty} A_{1}^{-i} A_{2}^{-j} \mathcal{C}$ is the maximal such subspace.

Proposition 2.2: The maximal $\left(A_{1}, A_{2}\right)$-invariant subspace included in $\mathcal{C}$ is

$$
\max I\left(A_{1}, A_{2} ; \mathcal{C}\right)=\bigcap_{i=0}^{n-1} \bigcap_{j=0}^{n-1} A_{1}^{-i} A_{2}^{-j} \mathcal{C}
$$

Proof: Let us denote by $\mathcal{V}$ the subspace in the right-hand member of (2.3). Obviously, by Proposition 2.1, $\mathcal{U} \subset \mathcal{V}$, where $\mathcal{U}=\bigcap_{i=0}^{\infty} \bigcap_{j=0}^{\infty} A_{1}^{-i} A_{2}^{-j} \mathcal{C}=\max I\left(A_{1}, A_{2} ; \mathcal{C}\right)$.

Now, for any $v \in \mathcal{V}, A_{1} A_{2}^{j} v \in \mathcal{C}, \forall i, j \in \mathbf{N}, i, j \leq n-1$. Let $p_{k}(s)=\operatorname{det}\left(s I-A_{k}\right)=s^{n}+a_{n-1, k} s^{n-1}+\cdots+a_{1, k} s+$ $a_{0, k}, k=1,2$ be the characteristic polynomial of the matrix $A_{k}, \quad k=1,2$. By Hamilton-Cayley Theorem, each matrix verifies its characteristic equation, hence

$A_{k}^{n}=-a_{n-1, k} A_{k}^{n-1}-\cdots-a_{1, k} A_{k}-a_{0, k} I, \quad k=1,2$.
Then, for any vector $v \in \mathcal{V}, A_{1}^{n} v=-\sum_{i=0}^{n-1} a_{i, k} A_{1}^{i} v$. Since $A_{1}$ and $A_{2}$ are commutative matrices, we can premultiply this equality by $A_{2}^{j}$ and we obtain $A_{1}^{n} A_{2}^{j} v=-\sum_{i=0}^{n-1} a_{i, k} A_{1}^{i} A_{2}^{j} v$ forall $j, 0 \leq j \leq n-1$. Since $\mathcal{C}$ is a subspace, one obtains $A_{1}^{n} A_{2}^{j} v \in \mathcal{C}$. Similarly, by applying recurrently the HamiltonCayley Theorem for both $A_{1}$ and $A_{2}$, we get $A_{1}^{i} A_{2}^{j} v \in$ $\mathcal{C}, \forall i, j \geq 0$, hence $v \in \mathcal{U}$. One obtains $\mathcal{V} \subset \mathcal{U}$, hence $\mathcal{V}=\mathcal{U}=\max I\left(A_{1}, A_{2} ; \mathcal{C}\right)$.

The following algorithm determines recurrently the subspace $\max I\left(A_{1}, A_{2} ; \mathcal{C}\right)$.

\section{Algorithm 2.1}

Stage 1. Construct the sequence of subspaces $\left(S_{0, j}\right)_{0 \leq j \leq n}$ of the space $\mathbf{K}^{n}$ :

$$
\begin{array}{r}
S_{0,0}=\mathcal{C} ; \\
S_{0, j}=\mathcal{C} \cap A_{2}^{-1} S_{0, j-1}, j=1, \ldots, n ;
\end{array}
$$

Stage 2. Determine $j_{0}$, the first index in $\{0,1, \ldots, n-1\}$ which verifies

$$
S_{0, j_{0}+1}=S_{0, j_{0}} \text {. }
$$

If $j_{0}=n-1$, then $\max I\left(A_{1}, A_{2} ; \mathcal{C}\right)=\{0\}$. STOP

If $j_{0}<n-1$, GO TO Stage 3 .

Stage 3. Construct the sequence of subspaces $\left(S_{i, j_{0}}\right)_{0 \leq i \leq n}$ :

$$
S_{i, j_{0}}=S_{i-1, j_{0}} \cap A_{1}^{-1} S_{i-1, j_{0}} .
$$

Stage 4. Determine $i_{0}$, the first index in $0,1, \ldots, n-1$ which verifies

$$
S_{i_{0}+1, j_{0}}=S_{i_{0}, j_{0}} .
$$

Then $\max I\left(A_{1}, A_{2} ; \mathcal{C}\right)=S_{i_{0}, j_{0}}$. STOP

Proof: Let us consider the doubly-indexed sequence of subspaces of the space $\mathbf{K}^{n}$

$$
\widetilde{S}_{i, j}=\left(\bigcap_{k=0}^{i} A_{1}^{-k}\right)\left(\bigcap_{l=0}^{j} A_{2}^{-l}\right) \mathcal{C}, i, j \in\{0,1, \ldots, n\} .
$$

By Proposition 2.1, $\max I\left(A_{1}, A_{2} ; \mathcal{C}\right) \subseteq \widetilde{S}_{i, j} \forall i, j$ and by Proposition 2.2, $\widetilde{S}_{n-1, n-1}=\max I\left(A_{1}, A_{2} ; \mathcal{C}\right)$. Obviously

$$
\widetilde{S}_{i, j} \supseteq \widetilde{S}_{k, l}, \forall k \geq i, l \geq j
$$

and $\widetilde{S}_{0,0}=A_{1}^{-0} A_{2}^{-0} \mathcal{C}=\mathcal{C}=S_{0,0}$. For any $j \in\{0,1, \ldots, n\}$, $\widetilde{S}_{0, j}=\cap_{l=0}^{j} A_{2}^{-l} \mathcal{C}=\mathcal{C} \cap A_{2}^{-1}\left(\cap_{l=0}^{j-1} A_{2}^{-l} \mathcal{C}\right)=\mathcal{C} \cap A_{2}^{-1} \widetilde{S}_{0, j-1}$.

Then, if we assume that $\widetilde{S}_{0, j-1}=S_{0, j-1}$, using (2.6) we get by induction the following equality:

$$
\widetilde{S}_{0, j}=S_{0, j}, \forall j \in\{0,1, \ldots, n\},
$$

hence by (2.11)

$$
S_{0, j} \supseteq S_{0, j+1}, \forall j \in\{0,1, \ldots, n-1\},
$$

Using again Hamilton-Cayley Theorem (see (2.4)) and (2.13) one obtains $S_{0, n}=\widetilde{S}_{0, n}=\widetilde{S}_{0, n-1}=S_{0, n-1}$, hence $j_{0} \leq n-1$. 
Let us consider the chain of subspaces

$\{0\} \subseteq S_{0, n}=S_{0, n-1} \subseteq S_{0, n-2} \subseteq \ldots \subseteq S_{0,1} \subseteq S_{0,0} \subseteq \mathbf{K}^{n}$.

Since $\mathcal{C}$ is a proper subspace of $X=\mathbf{K}^{n}$ it results that $\operatorname{dim} S_{0,0}=\operatorname{dim} \mathcal{C} \leq n-1$. If $j_{0}=n-1$ is the first index which verifies (2.7), we have

$0 \leq \operatorname{dim} S_{0, n-1}<\operatorname{dim} S_{0, n-2}<\cdots<\operatorname{dim} S_{0,0} \leq n-1$

hence $\operatorname{dim} S_{0, n-1}=0$, i.e. $S_{0, n-1}=\{0\}$. Therefore one obtains by Proposition $2.1\{0\}=S_{0, n-1}=\widetilde{S}_{0, n-1} \supseteq$ $\max I\left(A_{1}, A_{2} ; \mathcal{C}\right)$, hence $\max I\left(A_{1}, A_{2} ; \mathcal{C}\right)=\{0\}$.

If $j_{0}<n-1$ one obtains by $S_{0, j_{0}+1}=S_{0, j_{0}}$ and by (2.6) $S_{0, j_{0}+2}=\mathcal{C} \cap A_{2}^{-1} S_{0, j_{0}+1}=\mathcal{C} \cap A_{2}^{-1} S_{0, j_{0}}=S_{0, j_{0}+1}=S_{0, j_{0}}$.

Let us assume that $S_{0, j}=S_{0, j_{0}}$ for some $j \in\left\{j_{0}+1, j_{0}+\right.$ $2, \ldots, n-1\}$. Then, again by (2.6), $S_{0, j+1}=\mathcal{C} \cap A_{2}^{-1} S_{0, j}=$ $\mathcal{C} \cap A_{2}^{-1} S_{0, j_{0}}=S_{0, j_{0}+1}=S_{0, j_{0}}$ hence

$$
S_{0, j}=S_{0, j_{0}}, \forall j \in\left\{j_{0}+1, j_{0}+2, \ldots, n\right\} .
$$

Now, let us assume that $\widetilde{S}_{i-1, j_{0}}=S_{i-1, j_{0}}$, for some $i \in\{1,2, \ldots, n-1\}$. We use the obvious equality $\mathcal{V}_{1} \cap \mathcal{V}_{2} \cap$ $\mathcal{V}_{3}=\left(\mathcal{V}_{1} \cap \mathcal{V}_{2}\right) \cap\left(\mathcal{V}_{2} \cap \mathcal{V}_{3}\right)$, where $\mathcal{V}_{k}, k=1,2,3$ are any subspaces of $\mathbf{K}^{n}$. By (2.10) and (2.8) one obtains:

$\widetilde{S}_{i, j_{0}}=\cap_{k=0}^{i} \cap_{l=0}^{j_{0}} A_{1}^{-k} A_{2}^{-l} \mathcal{C}=\left(\cap_{k=0}^{i-1} \cap_{l=0}^{j_{0}} A_{1}^{-k} A_{2}^{-l} \mathcal{C}\right) \cap$ $\left(\cap_{k=1}^{i} \cap_{l=0}^{j_{0}} A_{1}^{-\bar{k}} A_{2}^{-l} \mathcal{C}\right)=\left(\cap_{k=0}^{i-1} \cap_{l=0}^{j_{0}} A_{1}^{-k} A_{2}^{-l} \mathcal{C}\right) \cap$ $A_{1}^{-1}\left(\cap_{k=0}^{i-1} \cap_{l=0}^{j_{0}} A_{1}^{-k} A_{2}^{-l} \mathcal{C}\right)=\widetilde{S}_{i-1, j_{0}} \cap A_{1}^{-1} \widetilde{S}_{i-1, j_{0}}=$ $S_{i-1, j_{0}} \cap A_{1}^{-1} S_{i-1, j_{0}}=S_{i, j_{0}}$, hence $\widetilde{S}_{i, j_{0}}=S_{i, j_{0}}, \forall i \in$ $\{0,1, \ldots, n\}$. It follows by (2.11) that $S_{i, j_{0}} \supseteq S_{i+1, j_{0}}$ and by Hamilton-Cayley Theorem that $S_{n-1, j_{0}}=S_{n, j_{0}}$.

Now, let us consider the chain of subspaces

$$
\{0\} \subseteq S_{n, j_{0}}=S_{n-1, j_{0}} \subseteq S_{n-2, j_{0}} \subseteq \cdots \subseteq S_{0, j_{0}} \subseteq \mathbf{K}^{n} .
$$

Since $\operatorname{dim} S_{0, j_{0}} \leq \operatorname{dim} S_{0,0} \leq n-1$, if $i_{0}=n-1$ we obtain as above that $\operatorname{dim} S_{n-1, j_{0}}=0$, hence $S_{n-1, j_{0}}=$ $\max I\left(A_{1}, A_{2} ; \mathcal{C}\right)=\{0\}$.

If $i_{0}<n-1$, we have by $\widetilde{S}_{i_{0}+1, j_{0}}=S_{i_{0}+1, j_{0}}=S_{i_{0}, j_{0}}=$ $\widetilde{S}_{i_{0}, j_{0}}$ and by (2.9) the following equalities: $\widetilde{S}_{i_{0}+2, j_{0}}=$ $\widetilde{S}_{i_{0}+1, j_{0}} \cap A_{1}^{-1} \widetilde{S}_{i_{0}+1, j_{0}}=\widetilde{S}_{i_{0}, j_{0}} \cap A_{1}^{-1} \widetilde{S}_{i_{0}, j_{0}}=\widetilde{S}_{i_{0}+1, j_{0}}=$ $\widetilde{S}_{i_{0}, j_{0}}$.

If we assume that $\widetilde{S}_{i, j_{0}}=\widetilde{S}_{i_{0}, j_{0}}$ for some $i \in\left\{i_{0}+\right.$ $\left.1, i_{0}+2, \ldots, n-1\right\}$, we get again by (2.9) $\widetilde{S}_{i+1, j_{0}}=$ $\widetilde{S}_{i, j_{0}} \cap A_{1}^{-1} \widetilde{S}_{i, j_{0}}=\widetilde{S}_{i_{0}, j_{0}} \cap A_{1}^{-1} \widetilde{S}_{i_{0}, j_{0}}=\widetilde{S}_{i_{0}+1, j_{0}}=\widetilde{S}_{i_{0}, j_{0}}$, hence

$$
\widetilde{S}_{i, j_{0}}=S_{i_{0}, j_{0}}, \forall i \in\left\{i_{0}+1, i_{0}+2, \ldots, n-1\right\} .
$$

Using (2.9), (2.14) and (2.15) we obtain $\cap_{l=0}^{j} A_{2}^{-l} \mathcal{C}=$ $\cap_{l=0}^{j_{0}} A_{2}^{-l} \mathcal{C}, \forall j \in\left\{j_{0}+1, j_{0}+2, \ldots, n\right\}$ and $\cap_{k=0}^{i} \cap_{l=0}^{j}$ $A_{1}^{-k} A_{2}^{-l} \mathcal{C}=\cap_{k=0}^{i} \cap_{l=0}^{j_{0}} A_{1}^{-k} A_{2}^{-l} \mathcal{C} \forall i \in\left\{i_{0}+1, i_{0}+2, \ldots, n\right\}$.

Then, $\forall i \in\left\{i_{0}+1, i_{0}+2, \ldots, n\right\}$ and $\forall j \in\left\{j_{0}+\right.$ $\left.1, j_{0}+2, \ldots, n\right\}$, one obtains $\widetilde{S}_{i, j}=\cap_{k=0}^{i} \cap_{l=0}^{j} A_{1}^{-k} A_{2}^{-l} \mathcal{C}=$ $\cap_{k=0}^{i} A_{1}^{-k}\left(\cap_{l=0}^{j} A_{2}^{-l} \mathcal{C}\right)=\cap_{k=0}^{i} A_{1}^{-k}\left(\cap_{l=0}^{j_{0}} A_{2}^{-l} \mathcal{C}\right)=$ $\cap_{k=0}^{i_{0}} \cap_{l=0}^{j_{0}} A_{1}^{-k} A_{2}^{-l} \mathcal{C}=S_{i_{0}, j_{0}}$, hence $\widetilde{S}_{i, j}=S_{i_{0}, j_{0}}, \forall i \in$ $\left\{i_{0}+1, i_{0}+2, \ldots, n\right\}, \forall j \in\left\{j_{0}+1, j_{0}+2, \ldots, n\right\}$.

By Proposition 2.2, we obtain

$$
\max I\left(A_{1}, A_{2} ; \mathcal{C}\right)=\widetilde{S}_{n-1, n-1}=S_{i_{0}, j_{0}},
$$

which completes the proof of the algorithm.

Given the $n \times n$ matrices A1, A2 and the $n \times k$ matrix Cal, the next Matlab program calculates an orhonormal basis in the maximal subspace $\max I(A 1, A 2 ; \mathrm{Cal})$ and its dimension:

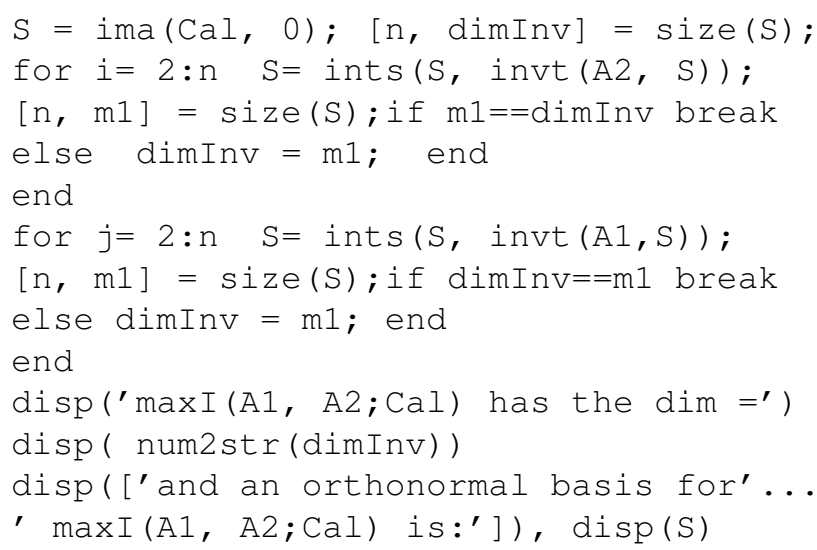

This program will be furher adapted for the algorithm in the last section and a concrete example will be given there.

From (2.10), (2.16) and $\widetilde{S}_{i_{0}, j_{0}}=S_{i_{0}, j_{0}}$ one obtains:

Proposition 2.3: The maximal $\left(A_{1}, A_{2}\right)$-invariant subspace included in the subspace $\mathcal{C}$ is

$$
\max I\left(A_{1}, A_{2} ; \mathcal{C}\right)=\widetilde{S}_{i_{0}, j_{0}}=\bigcap_{i=0}^{i_{0}} \bigcap_{j=0}^{j_{0}} A_{1}^{-i} A_{2}^{-j} \mathcal{C}
$$

\section{THE STATE SPACE REPRESENTATION OF THE 2D HYBRID SYSTEMS}

The linear spaces $X=\mathbf{R}^{n}, U=\mathbf{R}^{m}$ and $Y=\mathbf{R}^{p}$, are called respectively the state, input and output spaces and $T=$ $\mathbf{R} \times \mathbf{Z}$ is the time set. By $(s, l) \leq(t, k)$ for $(s, l),(t, k) \in T$ we mean $s \leq t, l \leq k$ and $(s, l)<(t, k)$ means $(s, l) \leq(t, k)$ and $(s, l) \neq(t, k)$.

Definition 3.1: A two-dimensional hybrid (continuousdiscrete) linear system (2Dcd) is a quintuplet

$\Sigma=\left(A_{1}(t, k), A_{2}(t, k), B(t, k), C(t, k), D(t, k)\right) \quad \in$ $\mathbf{R}^{n \times n} \times \mathbf{R}^{n \times n} \times \mathbf{R}^{n \times m} \times \mathbf{R}^{p \times n} \times \mathbf{R}^{p \times m}$ with $A_{1}(t, k) A_{2}(t, k)=A_{2}(t, k) A_{1}(t, k) \forall(t, k) \in T$, where all matrices are continuous with respect to $t \in \mathbf{R}$ for any $k \in \mathbf{Z}$; the state space representation of $\Sigma$ is given by the following state and output equations (where $\dot{x}(t, k)=\frac{\partial x}{\partial t}(t, k)$ ).

$$
\begin{array}{r}
\dot{x}(t, k+1)=A_{1}(t, k+1) x(t, k+1)+A_{2}(t, k) \dot{x}(t, k) \\
-A_{1}(t, k) A_{2}(t, k) x(t, k)+B(t, k) u(t, k) \\
y(t, k)=C(t, k) x(t, k)+D(t, k) u(t, k) .
\end{array}
$$

The vectors $x(t, k) \in X, u(t, k) \in U$ and $y(t, k) \in Y$ are respectively the state, the input and the output of the system $\Sigma$ at the moment $(t, k) \in T$.

We denote by $\Phi\left(t, t_{0} ; k\right)$ or $\Phi_{A_{1}}\left(t, t_{0} ; k\right)$ the continuous fundamental matrix of $A_{1}(t, k)$ with respect to $t \in \mathbf{R}$, for any fixed $k \in \mathbf{Z} . \Phi\left(t, t_{0} ; k\right)$ has the following properties, for 
any $t, t_{0}, t_{1} \in \mathbf{R}$ :

$$
\begin{array}{rc}
\text { i) } & \frac{\mathrm{d}}{\mathrm{d} t} \Phi\left(t, t_{0} ; k\right)=A_{1}(t, k) \Phi\left(t, t_{0} ; k\right), \\
\text { ii) } & \Phi\left(t_{0}, t_{0} ; k\right)=I_{n}, \\
\text { iii) } & \Phi\left(t, t_{1} ; k\right) \Phi\left(t_{1}, t_{0} ; k\right)=\Phi\left(t, t_{0} ; k\right), \\
\text { iv) } & \Phi\left(t, t_{0} ; k\right)^{-1}=\Phi\left(t_{0}, t ; k\right),
\end{array}
$$

$$
\begin{array}{r}
v) \Phi\left(t, t_{0} ; k\right)=I+\sum_{l=1}^{\infty} \int_{t_{0}}^{t} A_{1}\left(s_{1}, k\right) \int_{t_{0}}^{s_{1}} A_{1}\left(s_{2}, k\right) \cdots \\
\cdots \int_{t_{0}}^{s_{l-1}} A_{1}\left(s_{l}, k\right) \mathrm{d} s_{l} \mathrm{~d} s_{l-1} \cdots \mathrm{d} s_{2} \mathrm{~d} s_{1} .
\end{array}
$$

If $A_{1}$ is a constant matrix, then $\Phi\left(t, t_{0} ; k\right)=$ $\sum_{l=0}^{\infty} \frac{A_{1}^{l}\left(t-t_{0}\right)^{l}}{l !}=e^{A_{1}\left(t-t_{0}\right)}$.

We denote by $F\left(t ; k, k_{0}\right)$ the discrete fundamental matrix of the matrix $A_{2}(t, k)$, defined by

$$
\begin{aligned}
& F\left(t ; k, k_{0}\right)= \\
& =\left\{\begin{array}{cl}
A_{2}(t, k-1) A_{2}(t, k-2) \cdots A_{2}\left(t, k_{0}\right) & \text { for } k>k_{0} \\
I_{n} & \text { for } k=k_{0}
\end{array}\right.
\end{aligned}
$$

for any fixed $t \in \mathbf{R}$.

If $A_{2}$ is a constant matrix, then $F\left(t ; k, k_{0}\right)=A_{2}^{k-k_{0}}$.

The matrices $\Phi\left(t, t_{0} ; k\right)$ and $F\left(s ; l, l_{0}\right)$ commute for any $t, t_{0}, s \in \mathbf{R}$ and $k, l, l_{0} \in \mathbf{Z}$ since $A_{1}(t, k)$ and $A_{2}(t, k)$ are commutative matrices.

Definition 3.2: A vector $x_{0} \in X$ is called the initial state of $\Sigma$ at the moment $\left(t_{0}, k_{0}\right) \in T$ if, for any $(t, k) \in T$ with $(t, k) \geq\left(t_{0}, k_{0}\right)$ the following conditions hold:

$$
x\left(t, k_{0}\right)=\Phi\left(t, t_{0} ; k_{0}\right) x_{0}, x\left(t_{0}, k\right)=F\left(t_{0} ; k, k_{0}\right) x_{0} .
$$

For $\left(t_{0}, k_{0}\right) \leq(t, k)$ we denote by $\left[t_{0}, t ; k_{0}, k\right]$ the set $\left[t_{0}, t ; k_{0}, k\right]=\left[t_{0}, t\right] \times\left\{k_{0}, k_{0}+1, \ldots, k\right\}$.

From [15, Proposition 2.3] we obtain:

Proposition 3.1: The state of the system $\Sigma$ at the moment $(t, k) \in T$ determined by the control $u(\cdot, \cdot):\left[t_{0}, t ; k_{0}, k\right] \rightarrow U$ and by the initial state $x_{0} \in X$ is

$$
\begin{array}{r}
x(t, k)=\Phi\left(t, t_{0} ; k\right) F\left(t_{0} ; k, k_{0}\right) x_{0}+ \\
\int_{t_{0}}^{t} \sum_{l=k_{0}}^{k-1} \Phi(t, s ; k) F(s ; k, l+1) B(s, l) u(s, l) \mathrm{d} s .
\end{array}
$$

By replacing the state $x(t, k)$ given by (3.4) in the output equation (3.2) we get

Proposition 3.2: The input-output map of the system $\Sigma$ is given by the formula

$$
\begin{array}{r}
y(t, k)=C(t, k) \Phi\left(t, t_{0} ; k\right) F\left(t_{0} ; k, k_{0}\right) x_{0}+ \\
+\int_{t_{0}}^{t} \sum_{l=k_{0}}^{k-1} C(t, k) \Phi(t, s ; k) F(s ; k, l+1) B(s, l) u(s, l) \mathrm{d} s+
\end{array}
$$

\section{OBSERVABILITY OF TIME-VARYING 2D SYSTEMS}

In this section we present the study of the observability of the time-varying system $\Sigma=$ $\left(A_{1}(t, k), A_{2}(t, k), B(t, k), C(t, k), D(t, k)\right)$, along the line of [16].

A triplet $(t, k, \tilde{x}) \in T \times X$ is called a phase of $\Sigma$ if $\tilde{x}=$ $x(t, k)$ (i.e. $\tilde{x}$ is the state of $\Sigma$ at the moment $(t, k)$ ). One obtains by (3.4) that $(t, k, \tilde{x})$ is a phase of $\Sigma$ iff there exist a control $u(\cdot, \cdot)$ and an initial state $x_{0} \in X$ such that

$$
\begin{array}{r}
\tilde{x}=\Phi\left(t, t_{0} ; k\right) F\left(t_{0} ; k, k_{0}\right) x_{0}+ \\
+\int_{t_{0}}^{t} \sum_{l=k_{0}}^{k-1} \Phi(t, s: k) F(s ; k, l+1) B(s, l) u(s, l) \mathrm{d} s .
\end{array}
$$

We denote by $I$ the set $I=\left[t_{0}, t\right] \times\left[k_{0}, k\right] \subset T$, where $\left(t_{0}, k_{0}\right),(t, k) \in T$ and $\left(t_{0}, k_{0}\right)<(t, k)$.

Definition 4.1: A phase $\left(t_{0}, k_{0}, x\right)$ is said to be unobservable (unobservable on $I$ ) if for any control $u(\cdot, \cdot)$ it provides the same output $y(s, l)$ for $(s, l) \geq\left(t_{0}, k_{0}\right)$ (for $(s, l) \in I$ ) as the phase $\left(t_{0}, k_{0}, 0\right)$.

A state $x \in X$ is said to be unobservable (unobservable on $I)$ if there exists $\left(t_{0}, k_{0}\right) \in T$ such that the phase $\left(t_{0}, k_{0}, x\right)$ is unobservable (unobservable on $I$ ).

Proposition 4.1: The phase $\left(t_{0}, k_{0}, x\right)$ is unobservable if and only if

$$
C(t, k) \Phi\left(t, t_{0} ; k\right) F\left(t_{0} ; k, k_{0}\right) x=0
$$

for any $(t, k) \in T,(t, k) \geq\left(t_{0}, k_{0}\right)$.

The phase $\left(t_{0}, k_{0}, x\right)$ is unobservable on $I$ if and only if

$$
C(s, l) \Phi\left(s, t_{0} ; l\right) F\left(t_{0} ; l, k_{0}\right) x=0
$$

for any $(s, l) \in I$.

Proof: The output produced by the initial state $x$ given by (3.5) is denoted $y_{x}(t, k)$. If we replace $x_{0}$ by 0 in (3.5) we get the zero-input response of $\Sigma$

$$
\begin{array}{r}
y_{0}(t, k)=\int_{t_{0}}^{t} \sum_{l=k_{0}}^{k-1} C(t, k) \Phi(t, s: k) F(s ; k, l+1) B(s, l) . \\
\cdot u(s, l) \mathrm{d} s+D(t, k) u(t, k) .
\end{array}
$$

By equalizing $y_{x}(t, k)$ and $y_{0}(t, k)$ we get (4.2) ((4.3) if it is restricted to the interval $I$ ).

It results from (4.2) that the property of observability does not involve the matrices $B(t, k)$ and $D(t, k)$ : therefore in the sequel we shall often consider the system $\Sigma$ reduced to the triplet $\Sigma=\left(A_{1}(t, k), A_{2}(t, k), C(t, k)\right)$

Definition 4.2: The system $\Sigma$ is said to be completely observable (completely observable on $I$ ) if there is no unobservable (unobservable on $I$ ) state $x \neq 0$ 
In order to check whether a system $\Sigma$ is completely observable we introduce the 2D observability Gramian of $\Sigma$ denoted by $\mathcal{O}_{\Sigma}\left(t, t_{0} ; k, k_{0}\right)$

$$
\begin{array}{r}
\mathcal{O}_{\Sigma}\left(t, t_{0} ; k, k_{0}\right)=\int_{t_{0}}^{t} \sum_{l=k_{0}}^{k} F\left(t_{0} ; l, k_{0}\right)^{T} \Phi\left(s, t_{0} ; l\right)^{T} . \\
\cdot C(s, l)^{T} C(s, l) \Phi\left(s, t_{0} ; l\right) F\left(t_{0} ; l, k_{0}\right) \mathrm{d} s .
\end{array}
$$

It results that $\mathcal{O}_{\Sigma}\left(t, t_{0} ; k, k_{0}\right)$ is a symmetrical, positive semidefinite $n \times n$ matrix.

Proposition 4.2: The phase $\left(t_{0}, k_{0}, x\right)$ (the state $x$ ) is unobservable on $I$ if and only if

$$
\mathcal{O}_{\Sigma}\left(t, t_{0} ; k, k_{0}\right) x=0 .
$$

Proof: Necessity. If the state $x$ is unobservable on $I$ then (4.3) holds for any $(s, l) \in I$, hence $\mathcal{O}_{\Sigma}\left(t, t_{0} ; k, k_{0}\right) x=$ $\int_{t_{0}}^{t} \sum_{l=k_{0}}^{k} F\left(t_{0} ; l, k_{0}\right)^{T} \Phi\left(s, t_{0} ; l\right)^{T} C(s, l)^{T} C(s, l) \Phi\left(s, t_{0} ; l\right)$. $\cdot F\left(t_{0} ; l, k_{0}\right) x \mathrm{~d} s=0$.

Sufficiency. If (4.5) holds, then $x^{T} \mathcal{O}_{\Sigma}\left(t, t_{0} ; k, k_{0}\right) x=$ 0 , equality which can be written as $\int_{t_{0}}^{t} \sum_{l=k_{0}}^{k}\left\|C(s, l) \Phi\left(s, t_{0} ; l\right) F\left(t_{0} ; l, k_{0}\right) x\right\|^{2} \mathrm{~d} s=0$. Since the integrand is non-negative, one obtains (4.3), hence by Proposition 4.1 the state $x$ is unobservable on $I$.

Corollary 4.1: The set of states which are unobservable on $I$ is the subspace $\operatorname{KerO}_{\Sigma}\left(t, t_{0} ; k, k_{0}\right)$.

Corollary 4.2: The phase $\left(t_{0}, k_{0}, x\right)$ is unobservable if and only if, for any $(t, k) \in T,(t, k) \geq\left(t_{0}, k_{0}\right)$, $\mathcal{O}_{\Sigma}\left(t, t_{0} ; k, k_{0}\right) x=0$.

Theorem 4.1: The system $\Sigma=\left(A_{1}(t, k), A_{2}(t, k), C(t, k)\right)$ is completely observable on $I$ if and only if

$$
\operatorname{rank} \mathcal{O}_{\Sigma}\left(t, t_{0} ; k, k_{0}\right)=n
$$

Proof: By Corollary 4.1, $\Sigma$ is completely observable on $I$ if and only if $\operatorname{KerO}_{\Sigma}\left(t, t_{0} ; k, k_{0}\right)=\{0\}$, condition which is equivalent to (4.6).

Now it is possible to determine the initial state by knowing the input and the output of $\Sigma$.

Theorem 4.2: Let $\Sigma$ be completely observable on I. If the control $u(s, l)$ produces the output $y(s, l),(s, l) \in I$, then the initial state $x_{0}$ is given by

$$
\begin{array}{r}
x_{0}=\mathcal{O}_{\Sigma}\left(t, t_{0} ; k, k_{0}\right)^{-1} \int_{t_{0}}^{t} \sum_{l=k_{0}}^{k} F\left(t_{0} ; l, k_{0}\right)^{T} . \\
\cdot \Phi\left(s, t_{0} ; l\right)^{T} C(s, l)^{T} \tilde{y}(s, l) \mathrm{d} s
\end{array}
$$

where

$$
\begin{array}{r}
\tilde{y}(s, l)=y(s, l)-\int_{t_{0}}^{s} \sum_{\lambda=k_{0}}^{l-1} C(s, l) \Phi(s, \tau ; l) . \\
\cdot F(\tau ; l, \lambda+1) B(\tau, \lambda) u(\tau, \lambda) \mathrm{d} \tau-D(s, l) u(s, l) .
\end{array}
$$

Proof: From (3.5) and (4.8) we obtain $C(s, l) \Phi\left(s, t_{0} ; l\right) F\left(t_{0} ; l, k_{0}\right) x_{0}=\tilde{y}(s, l)$. If we premultiply this equality by $F\left(t_{0} ; l, k_{0}\right)^{T} \Phi\left(s, t_{0} ; l\right)^{T} C(s, l)^{T}$, then we integrate on $\left[t_{0}, t\right]$ and we sum up for $l=\overline{k_{0}, k}$, we get

$$
\begin{array}{r}
\mathcal{O}_{\Sigma}\left(t, t_{0} ; k, k_{0}\right) x_{0}=\int_{t_{0}}^{t} \sum_{l=k_{0}}^{k} F\left(t_{0} ; l, k_{0}\right)^{T} . \\
. \Phi\left(s, t_{0} ; l\right)^{T} C(s, l)^{T} \tilde{y}(s, l) \mathrm{d} s
\end{array}
$$

Since $\Sigma$ is completely observable on $I$, by Theorem 4.1 the matrix $\mathcal{O}_{\Sigma}\left(t, t_{0} ; k, k_{0}\right)$ is nonsingular and (4.7) results by premultiplying (4.9) by its inverse.

Remark 4.1: By replacing $x_{0}$ given by (4.7) into (3.4) one can conclude that completely observable systems allow the reconstitution of the whole trajectory of the system $\Sigma$ $x(t, k),(t, k) \in T,(t, k) \geq\left(t_{0}, k_{0}\right)$.

\section{OBSERVABILITY OF TIME-INVARIANT HYBRID SYSTEMS}

The system $\Sigma$ given by Definition 3.1 is said to be timeinvariant (or stationnary) if $A_{1}, A_{2}, B, C, D$ are constant matrices. In this case we can consider the initial moment $\left(t_{0}, k_{0}\right)=(0,0)$.

Since for constant matrices $A_{1}$ and $A_{2}$ the two fundamental matrices become $\Phi(t, s ; 0)=e^{A_{1}(t-s)}$ and $F(0 ; k, l)=$ $A_{2}^{k-l},(t, k),(s, l) \in T^{+}:=\mathbf{R}^{+} \times \mathbf{Z}^{+},(t, k) \geq(s, l)$, the input-output map (3.5) can be written in the form

$$
\begin{array}{r}
y(t, k)=C e^{A_{1} t} A_{2}^{k} x_{0}+\int_{0}^{t} \sum_{l=0}^{k-1} C e^{A_{1}(t-s)} A_{2}^{k-l-1} . \\
\cdot B u(s, l) \mathrm{d} s+D u(t, k) .
\end{array}
$$

Definition 5.1: Two time-invariant systems $\Sigma=\left(A_{1}, A_{2}, B, C, D\right)$ and $\tilde{\Sigma}=\left(\tilde{A}_{1}, \tilde{A}_{2}, \tilde{B}, \tilde{C}, \tilde{D}\right)$ are said to be isomorphic if there exists a nonsingular matrix $T \in \mathbf{R}^{n \times n}$ such that

$$
\tilde{A}_{i}=T^{-1} A_{i} T, i=1,2 ; \tilde{B}=T^{-1} B ; \tilde{C}=C T ; \tilde{D}=D .
$$

These relations are the consequence of the change of basis $x=T \tilde{x}$ in the state space $X=\mathbf{R}^{n}$. Since $e^{\tilde{A}_{1} t}=T^{-1} e^{A_{1} t} T$ and $\tilde{A}_{2}^{k}=T^{-1} A_{2}^{k} T$, if we write the input-output maps of both systems $\Sigma$ and $\tilde{\Sigma}$ we get

Proposition 5.1: Two isomorphic systems have the same input-output map.

In the case of time-invariant systems, the observability Gramian (denoted by $\mathcal{O}(t, k)$ instead of $\mathcal{O}_{\Sigma}\left(t, t_{0} ; k, k_{0}\right)$ ) becomes

$$
\mathcal{O}(t, k)=\int_{0}^{t} \sum_{l=0}^{k}\left(A_{2}^{T}\right)^{l} e^{A_{1}^{T} s} C^{T} C e^{A_{1} s} A_{2}^{l} \mathrm{~d} s
$$

and Proposition 4.1 and Theorem 4.1 can be restated as 
Proposition 5.2: The state $x \in X$ is unobservable if and only if

$$
C e^{A_{1} t} A_{2}^{k} x=0
$$

for any $(t, k) \in T^{+}$.

Theorem 5.1: The system $\Sigma=\left(A_{1}, A_{2}, C\right)$ is completely observable if and only if

$$
\operatorname{rank} \mathcal{O}(t, k)=n
$$

for any $(t, k) \in T^{+}$.

Definition 5.2: The matrix

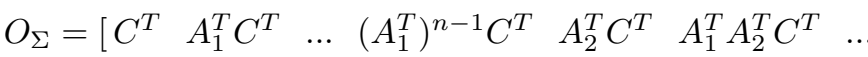

$$
\begin{aligned}
& \ldots\left(A_{1}^{T}\right)^{n-1} A_{2}^{T} C^{T} \quad \ldots \\
& \left.\left(A_{2}^{T}\right)^{n-1} C^{T} \quad A_{1}^{T}\left(A_{2}^{T}\right)^{n-1} C^{T} \ldots\left(A_{1}^{T}\right)^{n-1}\left(A_{2}^{T}\right)^{n-1} C^{T}\right]^{T} .
\end{aligned}
$$

is called the observability matrix of the system $\Sigma$.

Theorem 5.2: The system $\Sigma=\left(A_{1}, A_{2}, C\right)$ is completely observable if and only if

$$
\operatorname{rank} O_{\Sigma}=n
$$

Proof: Necessity. Let us assume that (5.3) fails, i.e. $\operatorname{rank} O_{\Sigma}<n$. Then there exists $x \in X \backslash\{0\}$, such that $O_{\Sigma} x=$ 0 . Then $C x=0, C A_{1} x=0, \ldots, C A_{1}^{n-1} A_{2}^{n-1} x=0$. By Hamilton-Cayley Theorem applied to $A_{1}$ and $A_{2}$ respectively (see ]2.4]), it results that $C A_{1}^{l} A_{2}^{k} x=0$ for any $l, k \in \mathbf{N}$. Then $C e^{A_{1} t} A_{2}^{k} x=C\left(\sum_{l=0}^{\infty} \frac{A_{1}^{l} t^{l}}{l !}\right) A_{2}^{k} x=\sum_{l=0}^{\infty} \frac{t^{l}}{l !} C A_{1}^{l} A_{2}^{k} x=0$. By Proposition $5.2 x$ is unobservable, hence $\Sigma$ is not completely observable.

Sufficiency. Let us suppose that $\Sigma$ is not completely observable. By Proposition 5.2 there exists $x \in X \backslash\{0\}$ such that $C e^{A_{1} t} A_{2}^{k} x=0$ for any $(t, k) \in T^{+}$. By deriving successively this equality with respect to $t$ and by taking $t=0$ we get $C A_{2}^{k} x=0, C A_{1} A_{2}^{k} x=0, \ldots, C A_{1}^{l} A_{2}^{k} x=0, \ldots, l, k \in \mathbf{N}$; this implies $O_{\Sigma} x=0$, hence $\operatorname{rank} O_{\Sigma}<n$.

The proof of Theorem 5.2 suggests the following result:

Theorem 5.3: The system $\Sigma=\left(A_{1}, A_{2}, C\right)$ is completely observable if and only if $C e^{A_{1} s} A_{2}^{l} x=0, \forall(s, l) \in[0, t] \times$ $[0, k] \subset T^{+}$for some $(t, k) \in T^{+}$implies $x=0$.

The following statement results from Theorem 5.2 and [15, Theorem 4.2], by noticing that the 2D observability matrix $O_{\Sigma}$ of the system $\Sigma=\left(A_{1}, A_{2}, B, C, D\right)$ coincides with the $2 \mathrm{D}$ controllability matrix $C_{\Sigma^{*}}$ of $\Sigma^{*}=\left(A_{1}^{T}, A_{2}^{T}, C^{T}, B^{T}, D^{T}\right)$. We say that the system $\Sigma^{*}$ is the dual of $\Sigma$.

Theorem 5.4: The system $\Sigma=\left(A_{1}, A_{2}, B, C, D\right)$ is completely observable if and only if its dual $\Sigma^{*}=$ $\left(A_{1}^{T}, A_{2}^{T}, C^{T}, B^{T}, D^{T}\right)$ is completely reachable.

Now we can give a characterization of the set of unobservable states, denoted by $X_{u o}$.
Proposition 5.3: The set $X_{u o}$ of all unobservable states of $\Sigma$ is the maximal $\left(A_{1}, A_{2}\right)$-invariant subspace of $X$ which is contained in $\operatorname{Ker} C$.

Proof: From the proof of Theorem 5.2 one can derive that $X_{u o}$ is the subspace $X_{u o}=\left\{x \in X \mid C A_{1}^{l} A_{2}^{k} x=0, \forall l, k \in\right.$ $\mathbf{N}\}$. Obviously, if $x \in X_{u o}$ then $l=k=0$ implies $x \in \operatorname{Ker} C$; moreover $A_{1} x \in X_{u o}$ since $C A_{1}^{l} A_{2}^{k}\left(A_{1} x\right)=$ $C A_{1}^{l+1} A_{2}^{k} x=0, \forall l, k \in \mathbf{N}$. Similarly $A_{2} x \in X_{u o}$ hence $X_{u o}$ is $\left(A_{1}, A_{2}\right)$-invariant and it is contained in $\operatorname{Ker} C$.

Now, let $\mathcal{V}$ be an $\left(A_{1}, A_{2}\right)$-invariant subspace of $X$ contained in $\operatorname{Ker} C$. Let $x$ be an element of $\mathcal{V}$. Since $\mathcal{V} \subset$ $\operatorname{Ker} C, C x=0 . \mathcal{V}$ being $\left(A_{1}, A_{2}\right)$-invariant we get $A_{1} x \in \mathcal{V}$ and $A_{2} x \in \mathcal{V}$ and by recurrence $A_{1}^{l} A_{2}^{k} x \in \mathcal{V} \subset \operatorname{Ker} C$, hence $C A_{1}^{l} A_{2}^{k} x=0, \forall l, k \in \mathbf{N}$, that is $x \in X_{u o}$. Therefore $\mathcal{V} \subset X_{u o}$ and $X_{u o}$ is the maximal such subspace of $X$.

By using Hamilton-Cayley Theorem for $A_{1}$ and $A_{2}$ (see[2.4]), we obtain $X_{u o}=\left\{x \in X \mid C A_{1}^{l} A_{2}^{k} x=0, \forall l, k=\right.$ $\overline{0, n-1}\}=\operatorname{Ker} O_{\Sigma}$. We proved:

Corollary 5.1: The set of all unobservable states of $\Sigma$ is $X_{u o}=\operatorname{Ker}_{\Sigma}$.

We obtain from Proposition 5.3:

Theorem 5.5: The system $\Sigma=\left(A_{1}, A_{2}, C\right)$ is completely observable if and only if $\{0\}$ is the greatest subspace of $X$ which is $\left(A_{1}, A_{2}\right)$-invariant and is contained in $\operatorname{Ker} C$.

The next result gives the canonical form of unobservable systems, byusing the geometric characterization of $X_{u o}$.

Theorem 5.6: The system $\Sigma=\left(A_{1}, A_{2}, C\right)$ is not completely observable if and only if it is isomorphic to a system $\tilde{\Sigma}=\left(\tilde{A}_{1}, \tilde{A}_{2}, \tilde{C}\right)$ with

$$
\tilde{A}_{i}=\left[\begin{array}{cc}
A_{11 i} & 0 \\
A_{21 i} & A_{22 i}
\end{array}\right], i=1,2, \quad \tilde{C}=\left[\begin{array}{cc}
C_{1} & 0
\end{array}\right],
$$

where $A_{11 i} \in \mathbf{R}^{\tilde{n} \times \tilde{n}}, C_{1} \in \mathbf{R}^{p \times \tilde{n}}$ and $\tilde{n}<n$. The triplet $\Sigma_{1}=\left(A_{111}, A_{112}, C_{1}\right)$ is completely observable.

Proof: We obtain the direct sum decomposition $X=X_{1} \oplus$ $X_{2}$ where $X_{2}=X_{u o} \neq\{0\}$ by considering a basis of $X_{2}$ and by completing it to a basis $\mathcal{B}$ of $X$. We denote by $\tilde{n}$ the dimension of the subspace $X_{1}$. Since $\Sigma$ is not completely observable $n-\tilde{n}=\operatorname{dim} X_{2}>0$, hence $\tilde{n}<n$.

In the basis $\mathcal{B}$ we have $X_{2}=\left\{x=\left[\begin{array}{c}0 \\ x_{2}\end{array}\right] \mid x_{2} \in \mathbf{R}^{n-\tilde{n}}\right\}$ and we denote the corresponding matrices of $\Sigma$ in this basis by $\tilde{A}_{1}, \tilde{A}_{2}$ and $\tilde{C}$. The transition matrix is denoted by $T$. Let us partition the matrix $\tilde{C}$ as $\tilde{C}=\left[\begin{array}{ll}C_{1} & C_{2}\end{array}\right]$ where $C_{1}$ has $\tilde{n}$ columns. By Proposition $5.3 X_{2}$ is included in $\operatorname{Ker} \tilde{C}$; then for any $x_{2} \in \mathbf{R}^{n-\tilde{n}}$ we have $\left[\begin{array}{ll}C_{1} & C_{2}\end{array}\right]\left[\begin{array}{c}0 \\ x_{2}\end{array}\right]=0$, i.e. $C_{2} x_{2}=0$, hence $C_{2}$ is a null matrix and $\tilde{C}=\left[\begin{array}{ll}C_{1} & 0\end{array}\right]$.

Since by Proposition $5.3 X_{2}$ is $\tilde{A}_{i}$-invariant, $i=1,2$, we have $\tilde{A}_{i}\left[\begin{array}{c}0 \\ x_{2}\end{array}\right] \in X_{2}$ for any $x_{2} \in \mathbf{R}^{n-\tilde{n}}$. Then if we partition $\tilde{A}_{i}$ as $\tilde{A}_{i}=\left[\begin{array}{cc}A_{11 i} & A_{12 i} \\ A_{21 i} & A_{22 i}\end{array}\right], i=1,2$ we 
obtain $\left[\begin{array}{c}A_{12 i} x_{2} \\ A_{22 i} x_{2}\end{array}\right]=\left[\begin{array}{c}0 \\ A_{22 i} x_{2}\end{array}\right]$ hence $A_{12 i} x_{2}=0$ for any $x_{2} \in \mathbf{R}^{n-\tilde{n}}$, i.e. $A_{12 i}=0, i=1,2$. Therefore in the basis $\mathcal{B}$ the system has the form (5.4).

By corollary $4.1 \tilde{n}=\operatorname{dim} X_{1}=n-\operatorname{dim} X_{2}=$ $n-\operatorname{dim} \operatorname{Ker} O_{\Sigma}=\operatorname{rank} O_{\Sigma}=\operatorname{rank} O_{\tilde{\Sigma}}$ since $O_{\tilde{\Sigma}}=$ $O_{\Sigma} T$ and $T$ is nonsingular. From (5.4) we get $\tilde{C} \tilde{A}_{i}^{k}=$ $\left[\begin{array}{cc}C_{1} A_{11 i}^{k} & 0\end{array}\right], \forall k \in \mathbf{Z}^{+}$. Then $O_{\tilde{\Sigma}}$ has the structure $O_{\tilde{\Sigma}}=$ $\left.\left[\begin{array}{ll}C_{1} A_{11 i}^{k} & 0\end{array}\right]\right]_{0 \leq k \leq n-1}$. By Hamilton-Cayley Theorem, for any $j \in \mathbf{N}, A_{11 i}^{\tilde{n}-1+j}$ is a linear combination of $A_{11 i}^{l}, l=$ $\overline{0, \tilde{n}-1}$, hence

$$
\begin{aligned}
\tilde{n}=\operatorname{rank} O_{\tilde{\Sigma}} & =\operatorname{rank}\left[\left[\begin{array}{ll}
C_{1} A_{11 i}^{k} & 0
\end{array}\right]\right]_{0 \leq k \leq \tilde{n}-1}= \\
& =\operatorname{rank}\left[C_{1} A_{11 i}^{k}\right]_{0 \leq k \leq \tilde{n}-1} ;
\end{aligned}
$$

But $\left[C_{1} A_{11 i}^{k}\right]_{0 \leq k \leq n-1}$ is the observability matrix $O_{\Sigma_{1}}$ of the system $\Sigma_{1}=\left(A_{111}, A_{112}, C_{1}\right)$. By Theorem 5.2, rank $O_{\Sigma_{1}}=$ $\tilde{n}$ implies the complete observability of $\Sigma_{1}$.

This Theorem can be restated as

Theorem 5.7: The system $\Sigma=\left(A_{1}, A_{2}, C\right)$ is completely observable if and only if it is not isomorphic to a system of the form (5.4).

Theorem 5.8: The system $\Sigma=\left(A_{1}, A_{2}, C\right)$ is completely observable if and only if there is no common eigenvector of the matrices $A_{1}$ and $A_{2}$ belonging to $\operatorname{Ker} C$.

Proof: Necessity. Let us assume that $\exists x \in \mathbf{R}^{n} \backslash\{0\}$ such that $A_{1} x=\lambda_{1} x, A_{2} x=\lambda_{2} x$ for some $\lambda_{1}, \lambda_{2} \in \mathbf{C}$ and $C x=$ 0 . Then $C A_{1}^{i} A_{2}^{j} x=\lambda_{1}^{i} \lambda_{2}^{j} C x=0$ for any $i, j \geq 0$; we get $O_{\Sigma} x=0$, hence $\operatorname{rank} O_{\Sigma}<n$ and by Theorem $5.2 \Sigma$ is not completely observable.

Sufficiency. Let us assume that $\Sigma$ is not completely observable. We denote by $S_{1}$ the subspace of $\mathbf{R}^{n} S_{1}=\operatorname{Ker} O_{\Sigma}$. Since $\operatorname{rank} O_{\Sigma}<n, \exists x \in \mathbf{R}^{n} \backslash\{0\}$ such that $O_{\Sigma} x=0$, hence $S_{1}$ contains nonzero vectors. For any $x \in S_{1}$ we get $C A_{1}^{i} A_{2}^{j} x=0, i, j=\overline{0, n-1}$ and by Hamilton-Cayley Theorem, applied to matrices $A_{1}$ and $A_{2}$, this equality is true $\forall i, j \geq 0$, hence $S_{1}=\left\{v \in \mathbf{R}^{n} \mid C A_{1}^{i} A_{2}^{j} x=0, \forall i, j \geq 0\right\}$.

We can prove that $S_{1}$ is $\left(A_{1}, A_{2}\right)$-invariant. Indeed, if $x \in S_{1}$, then for any $i, j \in \mathbf{N}$ we have $C A_{1}^{i} A_{2}^{j}\left(A_{1} x\right)=$ $C A_{1}^{i+1} A_{2}^{j} x=0$, hence $A_{1} x \in S_{1}$ and analogously $A_{2} x \in S_{1}$. Since $S_{1}$ is a proper $A_{1}$-invariant subspace of $\mathbf{R}^{n}$ it contains an eigenvector $x_{1}$ of $A_{1}$; let $\lambda$ be the corresponding eigenvalue of $A_{1}$. Let $S_{2}$ be the subspace $S_{2}=\left\{v \in \mathbf{R}^{n} \mid A_{1} v=\lambda v\right\}$; obviously $0 \neq x_{1} \in S_{2} . S_{2}$ is $A_{2}$-invariant since $x \in S_{2}$ implies $A_{1}\left(A_{2} x\right)=A_{2}\left(A_{1} x\right)=\lambda\left(A_{2} x\right)$, hence the subspace $S_{3}:=S_{1} \cap S_{2}$ is $A_{2}$-invariant and contains the nonzero vector $x_{1}$. It results that $S_{3}$ contains an eigenvector $x_{2}$ of $A_{2}$ which is also an eigenvector of $A_{1}$ since $S_{3} \subset S_{2}$. This eigenvector belongs to $\operatorname{Ker} O_{\Sigma}$ since $x_{2} \in S_{3} \subset S_{1}$ implies $O_{\Sigma} x=0$ and for $i=j=0$ we get $C x_{2}=0$.

A consequence of this Theorem is a Popov-HautusBelevitch type criterion of observability:
Theorem 5.9: The system $\Sigma=\left(A_{1}, A_{2}, C\right)$ is completely observable if and only if for any $s_{1}, s_{2} \in \mathbf{C}$

$$
\operatorname{rank}\left[\begin{array}{c}
C \\
s_{1} I-A_{1} \\
s_{2} I-A_{2}
\end{array}\right]=n .
$$

Proof: By Theorem 5.8 the statement that $\Sigma$ is not completely observable is equivalent to the existence of $x \in \mathbf{R}^{n}$ $\{0\}$ with $C x=0, A_{i} x=\lambda_{i} x, i=1,2$ for some $\lambda_{1}, \lambda_{2} \in \mathbf{C}$, that is such that $C x=0,\left(\lambda_{1} I-A_{1}\right) x=0,\left(\lambda_{2} I-A_{2}\right) x=$ $0, x \neq 0$; this is equivalent to the fact that the matrix in (4.7) has the rank less than $n$.

Since $\operatorname{rank}\left(s_{i} I-A_{i}\right)=n \quad \forall s_{i} \notin \sigma\left(A_{i}\right)$, Theorem 5.9 can be restated as

Corollary 5.2: The system $\Sigma=\left(A_{1}, A_{2}, C\right)$ is completely observable if and only if (5.5) holds for any $s_{1} \in \sigma\left(A_{1}\right), s_{2} \in$ $\sigma\left(A_{2}\right)$.

\section{THE DETERMinAtion OF THE SUBSPACE OF UNOBSERVABLE STATES}

Let us consider an LTI system $\Sigma=\left(A_{1}, A_{2}, C\right) \in$ $\mathbf{R}^{n \times n} \times \mathbf{R}^{n \times n} \times \mathbf{R}^{p \times n}$ with $A_{1}, A_{2}$ commutative matrices. We will adapt Algorithm 1 to determine the subspace $X_{u o}$ of unobservable states of the system $\Sigma$.

\section{Algorithm 6.1}

Stage 1. Determine the observability matrix $O_{\Sigma}$.

Stage 2. Compute $\operatorname{rank} O_{\Sigma}$.

If $\operatorname{rank} O_{\Sigma}=n$, then $X_{u o}=\{0\}$. STOP

If rank $O_{\Sigma}<n$ GO TO Stage 3 .

Stage 3. Construct the sequence of subspaces $\left(S_{0, j}\right)_{0 \leq j \leq n-2}$ of the space $X=\mathbf{R}^{n}$ :

$$
\begin{array}{r}
S_{0,0}=\operatorname{Ker} C \\
S_{0, j}=\operatorname{Ker} C \bigcap A_{2}^{-1} S_{0, j-1}, j=1, \ldots, n-2 .
\end{array}
$$

Stage 4 . Determine $j_{0}$, the first index in $\{0,1, \ldots, n-2\}$ which verifies

$$
S_{0, j_{0}+1}=S_{0, j_{0}} .
$$

Stage 5. Construct the sequence of subspaces $\left(S_{i, j_{0}}\right)_{0 \leq i \leq n-2}$ of the space $X=\mathbf{R}^{n}$ :

$$
S_{i, j_{0}}=S_{i-1, j_{0}} \bigcap A_{1}^{-1} S_{i-1, j_{0}} .
$$

Stage 6. Determine $i_{0}$, the first index in $\{0,1, \ldots, n-2\}$ which verifies

$$
S_{i_{0}+1, j_{0}}=S_{i_{0}, j_{0}} .
$$

Then $X_{u o}=S_{i_{0}, j_{0}}$. STOP

Proof: By Proposition 5.3, $X_{u o}=\max I\left(A_{1}, A_{2} ; \mathcal{C}\right)$ where $\mathcal{C}=\operatorname{Ker} C$.

If $\operatorname{rank} O_{\Sigma}=n$, then $\Sigma$ is completely observable (by Theorem 5.1), hence $X_{u o}=\{0\}$ (and in this case $i_{0}$ or $j_{0}$ 
would be equal to $n-1)$. Otherwise, $i_{0}<n-1, j_{0}<n-1$ and $\max I\left(A_{1}, A_{2} ; \mathcal{C}\right)=S_{i_{0}, j_{0}}$, hence $X_{\text {uo }}=S_{i_{0}, j_{0}}$.

The Matlab program presented below and based upon the algorithm above calculates an orthonormal basis of the unobservable states subspace and its dimension for the bidimensional case.

The instructions make use of the m-functions ima, ints and invt included in the Geometric Approach toolbox published by G. Marro and G. Basile at http://www3.deis.unibo.it/Staff/FullProf/GiovanniMarro/geometric.htm; this GA toolbox works with Matlab 5, Matlab 6 and Matlab 7 and the Control System Toolbox.

More precisely, given the matrices $A 1, A 2$ that commute and the matrix $C$, the next commands will compute and display the dimension and an orthonormal basis in the space $S=\max I(A 1, A 2 ; B)$.

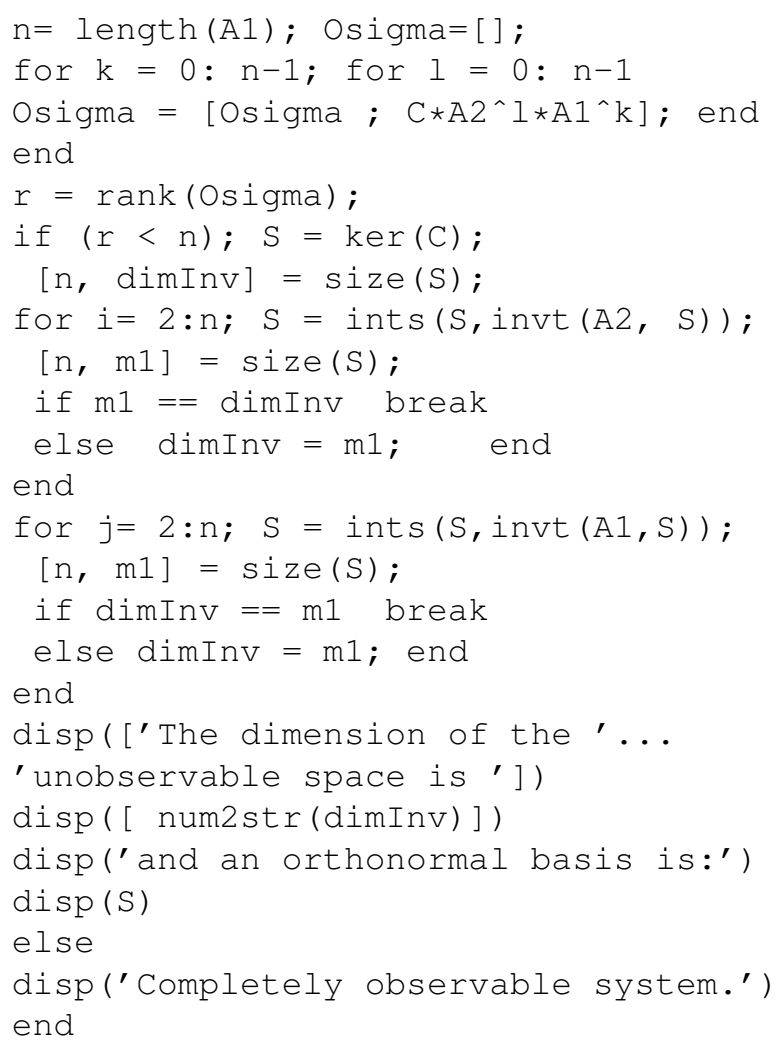

For example, given the matrices

$$
\begin{gathered}
A_{1}=\left[\begin{array}{llll}
3 & 0 & 0 & 0 \\
0 & 4 & 0 & 0 \\
0 & 0 & 0 & 0 \\
0 & 0 & 0 & 0
\end{array}\right], A_{2}=\left[\begin{array}{cccc}
0 & 0 & 0 & 0 \\
0 & 0 & 0 & 0 \\
0 & 0 & 2 & -1 \\
0 & 0 & -2 & 5
\end{array}\right], \\
C=\left[\begin{array}{cccc}
2 & 0 & 0 & 5 \\
-3 & 0 & 0 & -2
\end{array}\right],
\end{gathered}
$$

the above Matlab program gives the answers:

The dimension of the unobservable space is 1 and an orthonormal basis is:

$$
\mathcal{B}=\left\{\left[\begin{array}{llll}
0 & 1 & 0 & 0
\end{array}\right]^{T}\right\}
$$

\section{CONClusion}

The minimal subspace which is invariant with respect to some commutative matrices and which is included in a given subspace is determined by a suitable algorithm. This algorithm is applied to determine the subspace of the unobservable states of a hybrid 2D system. The state space representation of these systems is studied and observability criteria are obtained. Necessary and sufficient conditions of observability are derived for LTI 2D systems as well as the characterization of the unobservable states subspace.

These results and the proposed algorithms can be extended to $(q, r)$-D systems with $(q, r)>(1,1)$, i.e. to hybrid systems with $q$ continuous-time and $r$ discrete-time variables.

\section{REFERENCES}

[1] S. Attasi,'Introduction d'une classe de systèmes linéaires reccurents à deux indices", C.R. Acad. Sc. Paris, 277, 1973, 1135.

[2] G. Basile and G. Marro, "Controlled and Conditioned Invariants in Linear System Theory", J. Optim. Theory Appl., 3, 1969, 306-315.

[3] G. Basile and G. Marro, "L'invarianza rispetto ai disturbi studiata nello spazio degli stati”, Rendiconti della LXX Riunione Annuale AEI, paper 1.4.01, 1997.

[4] E. Fornasini and G. Marchesini, "State Space Realization Theory of Two-Dimensional Filters", IEEE Trans. Aut. Control, AC-21, 1976, 484-492.

[5] K. Galkovski, E. Rogers and D.H. Owens, "New 2D models and a transition matrix for discrete linear repetitive processes", Int. J. Control, 15,1999, 1365-1380.

[6] E.G. Gilbert, "Controllability and observability in multivariable control systems", SIAM J. Control, 1, 1963, 128-151.

[7] D.D. Givone and R.P. Roesser, "Minimization of multidimensional linear iterative circuits",IEEE Trans. Comput., C-22, 1973, 673-678.

[8] T. Kaczorek, "Controllability and minimum energy control of 2D continuous-discrete linear systems", Appl. Math. and Comp. Sci., 1, 1995, 5-21.

[9] R.E. Kalman, "Contributions to the theory of optimal contro". Proceedings of the Mexico City Conference on Ordinary Differential Equations, 1959.

[10] R.E. Kalman, P.L. Falb and M.A. Arbib, Topics in Mathematical System Theory. McGraw-Hill, 1969.

[11] S.-Y. Kung, B.C. Lévy, M. Morf and T. Kailath,'New Results in 2-D Systems Theory, Part II: 2-D State-Space Models-Realizations and the Notions of Controllability, Observability and Minimality", Proc. of the IEEE, 6, 1977, 945-961.

[12] J. Kurek and M.B. Zaremba,"Iterative learning control synthesis on 2D system theory", IEEE Trans. Aut. Control, 1, 1993, 121-125.

[13] G. Marro, Teoria dei sistemi e del controlo, Zanichelli, Bologna, 1989

[14] V. Prepeliţă, "Linear hybrid systems". Bull. Math. Soc. Sci. Math. de Roumanie, 4, 1979, 391-403.

[15] V. Prepeliţă, "Criteria of reachability for 2D continuous-discrete systems", Rev. Roumaine Math. Pures Appl., 48, 1, 2003, 81-93.

[16] V. Prepeliţă, Monica Pîrvan, "Observability of 2D continuous-discrete separable linear systems", Rev. Roumaine Math. Pures Appl., 48, 3, 2003, 283-297.

[17] R.P. Roesser, "A Discrete State-Space Model for Linear Image Processing", IEEE Trans. Aut. Control, 1, 1975, 1-10.

[18] L. Weiss, "Weighting Patterns and the Controllability and Observability of Linear Systems". Proc. Nat. Acad. of Sc., 51, 1964, 1122-1127.

[19] W. M. Wonham, "Linear Multivariable Control, a Geometric Approach, 2nd ed., Springer Verlag, New York, 1979.

\section{Creative Commons Attribution License 4.0 (Attribution 4.0 International, CC BY 4.0)}

This article is published under the terms of the Creative Commons Attribution License 4.0 https://creativecommons.org/licenses/by/4.0/deed.en_US 